\title{
DIVERSITY TECHNIQUES FOR BLIND CHANNEL EQUALIZATION IN MOBILE COMMUNICATIONS
}

\author{
Meritxell Lamarca, Gregori Vázquez \\ Dept. of Signal Theory and Communications, Polytechnic University of Catalonia (UPC) \\ c/Gran Capitán s/n, 08034-Barcelona (SPAIN); \\ Tel:+34-3-4016436, Fax: +34-3-4016447, E-mail: $\{x e l l$, gregori\}@gps.tsc.upc.es
}

\begin{abstract}
A blind algorithm for channel distortion compensation is presented which can be employed in spatial or temporal diversity receivers. The proposed technique can be used in frequency selective and frequency flat fading mobile channels, using burst transmission schemes in the first case and OFDM modulation in the second one. The algorithm is base on a deterministic criteria and is suited for estimation when short sets of data are available.
\end{abstract}

\section{INTRODUCTION}

In this paper a a blind technique is presented which can be employed for channel distortion compensation either in frequency flat or frequency selective mobile channels ([1]).

The proposed approach relies on the availability of space or time diversity which enables the use of single-input multiple-output formulation (SIMO) of the transmission system. It consists of an algorithm for linear equalization of the received data wich is based on a deterministic design criteria.

The suggested algorithm has a low computational load and exhibits a performance similar to that one of other deterministic criteria proposed in the literature: it obtains relatively good results for short data sets, it assumes the channel is FIR with known length (this constraint will be relaxed further on) and its original derivation does not take into account the additive noise, although it is of course considered when defining the method final formulation.

As opposed to methods which have appeared earlier in the literature, the proposed algorithm is based on the assumption that the receiver can observe the complete convolution of the transmitted data and the channel response. In the case of convolutive channels, the full channel output is available if a burst transmission scheme is employed and a guard time longer than channel response duration is inserted between consecutive transmitted frames. This is not a major constraint, given that this guard time is inserted in most of burst transmission schemes anyway.

In the case of multiplicative channels, the convolutive distortion arises when OFDM (Orthogonal Frequency Division Multiplexing) modulation ([2]) is applied. In [3] it was shown that OFDM and the transform modulations (defined in that paper) could improve BER performance in frequency flat fading channels. Also in [3], the simulations provided evidenced the improvements achieved when the received signal was equalized in the frequency domain. Here it will

\footnotetext{
${ }^{0}$ This work has been partially supported by the Spanish Re search Council (TIC-95-1022-C05-01, TIC-96-0500-C10-01) and by CIRIT/Generalitat de Catalunya (SGR0096)
}

be shown that the proposed algorithm is specially suited to blind equalization in this application of OFDM.

This paper extends the method proposed in [4], improving very significantly its robustness in front of the noise. The relationship of this algorithm with other methods proposed earlier in the literature is also explored.

\section{PROBLEM STATEMENT}

Figure (1a) shows a discrete-time model for a diversity receiver. The same information signal $T[k]$ is transmitted through $B$ diversity branches, it is distorted by different channel responses $C^{i}[k]$ and it is degraded by different additive white Gaussian noise terms $W^{i}[k]$. Using the $z$ transforms associated to these sequences, the received signal can be written as:

$$
Y^{i}(z)=T(z) \cdot C^{i}(z)+W^{i}(z) \quad i=1, \ldots, B
$$

The diagram in figure ( $1 \mathrm{a}$ ) is widely used for modelling the transmission through a convolutive channel when the desired diversity is achieved either by oversampling or using multiple antennas. It can also be employed for modelling the transmission of OFDM signals through frequency-flat fading channels when spatial diversity is employed at the receiver. In this case, the received signal can be expressed in the time domain as:

$$
y^{i}[n]=t[n] \cdot c^{i}[n]+w^{i}[n] \quad i=1, \ldots, B
$$

In the frequency domain, the multiplication distortion causes a spreading of the transmitted signal spectrum which appears in the received sequence as a convolution with the channel response, a circular one $(\circledast)$ in the case of working at 1 sample/symbol ([5]):

$$
Y^{i}[k]=T[k] \circledast C^{i}[k]+W^{i}[k] \quad i=1, \ldots, B
$$

or a linear one $(\circledast)$ when the received signal is oversampled $([4])$ :

$$
\begin{array}{rlr}
Y^{i}[k] & =T[k] * C^{i}[k]+W^{i}[k] & i=1, \ldots, B \\
Y^{i}(z) & =T(z) \cdot C^{i}(z)+W^{i}(z) & i=1, \ldots, B
\end{array}
$$

Since the mobile channel is slowly varying, the Doppler spread caused by the mobile channel is small compared to the transmitted signal bandwidth. Hence, the channel response $c^{i}[n]$ has only low-frequency components and, therefore, $C^{i}[k]$ can be regarded as a short duration sequence (see an example in fig.2). This means that the oversampling factor needed to have a linear convolution (or equivalently to satisfy Nyquist criterion) will be small (e.g. in the simulations in section 6 . was $135 / 128$ ). In the OFDM transmission through the $\mathrm{F}^{3}$-channel, from the channel compensation point of view, the advantage of working in the 
frequency domain is that the techniques developed for channel equalization in frequency-selective channels (linear and DFE equalization, blind algorithms, Maximum Likelihood estimation,etc.) can also be applied to the multiplicative channel.

Both in the frequency-selective and in the frequency flat cases, the receiver aims to retrieve the transmitted signal from the received data. In this paper, a linear equalizer is employed and a blind algorithm is proposed for estimating its coefficients. Notice that in mobile communications, when the vehicle speed is high, the channel response changes rapidly and a few data is be available to estimate the equalizer weights. In the multiplicative channel case this is even more evident, since the channel response $C^{i}[k]$ is completely different for each OFDM frame. The algorithm proposed in this paper is well suited for these applications.

Figure (1b) shows the linear equalization architecture employed in this paper. The multiple diversity branches are combined by means of FIR filters $E^{i}[k]$ to generate an output $R[k]$ :

$$
\begin{aligned}
R(z) & =\sum_{i=1}^{B} Y^{i}(z) \cdot E^{i}(z)= \\
& \left.=T(z) \cdot \sum_{i=1}^{B} C^{i}(z) \cdot E^{i}(z)+\sum_{i=1}^{B} W^{i}(z) \cdot E^{i}(z) 7\right)
\end{aligned}
$$

Thus, our problem can be stated as that one of designing the filters $E^{i}[k]$ in order to retrieve the transmitted data: $R[k]=T[k]$. The perfect equalization (zero forcing) criteria requires $R(z)=T(z)$ and therefore

$$
\sum_{i=1}^{B} C^{i}(z) \cdot E^{i}(z)=1
$$

In the next section a blind algorithm is summarized which provides the equalizer coefficients $E^{i}[k]$.

\section{BLIND CRITERIA}

The proposed algorithm is based on the observation that if the noise term is negligible

$$
Y^{i}(z)=T(z) \cdot C^{i}(z) \quad i=1, \ldots, B
$$

and therefore

$$
T(z)=\operatorname{g.c.d} .\left\{Y^{i}(z)\right\} \quad i=1, \ldots, B
$$

where g.c.d. stands for the greatest common divisor. Thus, the transmitted data can be estimated from $Y^{i}(z)$ using equation (10). Of course, in order to apply this equation the complete z-transform must be available and, therefore, in the case of a frequency selective channel a block transmission scheme with a guard time longer than the channel response is needed. On the contrary, in the multiplicative channel no efficiency is lost because the complete convolution is always observed if the received signals are properly sampled.

The proposed algorithm extracts the greatest common divisor resorting to the following property (Bezout equation) ([6]):

Given $B$ polynomials $\left\{A^{i}(z)\right\}_{i=1 \ldots B}$, the equation

$$
\sum_{i=1}^{B} A^{i}(z) \cdot a^{i}(z)=1
$$

has a solution in $a^{i}(z)$ iff the polynomials $A^{i}(z)$ are coprime. Furthermore, the solution is unique (up to a multiplicative constant) iff

$$
\operatorname{deg}\left\{a^{i}(x)\right\}+1=\frac{\operatorname{deg}\left\{A^{i}(x)\right\}}{B-1} \quad i=1, \ldots, B
$$

If the polynomials $a^{i}(x)$ have a greater degree, infinite solutions can be found for this equation.

When this property is applied to equation (8), it turns out that perfect channel equalization can be obtained only when channel responses have no common zeros, a result well known in the literature ([7]). Furthermore, from the previous property also follows that the zero-forcing equalizer is unique when the equalizer lengths are selected according to (12) and is non-unique if longer filters are applied, being the difference among the possible solutions their performance in front of the additive noise ([9]). Thus, designing longer equalizers allows for performance improvements in the Bit Error Rate (BER).

Besides, in the noiseless case, equation (6) states that the equalizer output $R(z)$ will always be a multiple of the transmitted data $T(z)$, and that

$$
\operatorname{deg}\{R(z)\}=\operatorname{deg}\{T(z)\}+\operatorname{deg}\left\{\sum_{i=1}^{B} C^{i}(z) \cdot E^{i}(z)\right\}
$$

Therefore, asking for an output of minimum length $(R(z)$ of minimum degree) is equivalent to asking for perfect channel equalization: $R[k]=T[k]$. This is the design criteria in which the proposed method is based: if $E^{i}[k]$ is designed so that $R[k]$ has minimum length, then $R[k]=\alpha T[k]$, being $\alpha$ an unknown complex constant. The matrix formulation for the method can be found in [4] and is briefly summarized here in order to introduce the new method.

As shown in [4], equation (6) can be written using matrix notation as

$$
\underline{R}=\underline{Y} \underline{E}
$$

where $\underline{R}$ is the equalizer output vector, $Y$ is a generalized Sylvester matrix with the received data and $\underline{E}$ is the equalizer weight vector. The perfect equalization case in (8) can be written as

$$
\underline{R}=\left[\frac{T}{\underline{\underline{0}}}\right] \alpha=\left[\begin{array}{l}
\underline{\underline{Y}}_{t} \\
\underline{\underline{\underline{Y}}}_{o}
\end{array}\right] \underline{E}
$$

where the received data matrix $\underline{\underline{Y}}$ has been split in two parts and $T$ is the transmitted data vector. The minimum length criteria can be described then as finding those equalizer coefficients $\underline{E}$ such that

$$
\underline{Q}=\underline{\underline{Y}} \underline{\underline{E}}
$$

Thus the method proposed in [4] is based on the noise subspace of matrix $\underline{\underline{Y}}_{0}$. Once the equalizer has been estimated, the received data can be filtered to yield an estimation of the transmitted data:

$$
\hat{\underline{T}}=\underline{\underline{Y}} \underline{\underline{E}} \underline{\hat{E}}
$$

\section{NEW ALGORITHM FORMULATION}

The previous method has two main drawbacks which are solved by the new approach proposed here:

- The previous algorithm can only be applied when equalizer lengths satisfy (12), for if they were overdimensioned the algorithm might converge to a non-useful 
solution with constant $\alpha=0$ and, therefore, $R[k]=0$. Hence, the advantages of long equalizers in terms of noise cannot be exploited.

- The previous algorithm does not fully exploit the available data. Both $\underline{Y}$ and $\underline{Y}$ contain information on the channel and the transmitted data, but the algorithm described in [4] designs the equalizer taps based on $\underline{\underline{Y}}$ only.

According to these considerations, the new algorithm formulation tries to maximize the Signal-to-ISI-plus-noise-ratio (SINR) at the equalizer output. This SINR can be approximately estimated (see eq. (15)) as

$$
\widehat{S I N R}=\frac{\underline{E}^{H} \underline{\underline{Y}}_{t}^{H} \underline{\underline{Y}} t \underline{\underline{E}}}{\underline{\underline{E}}^{H} \underline{\underline{Y}}_{0}^{H} \underline{\underline{Y}}_{0} \underline{\underline{E}}}
$$

This is the new cost function to be optimized. Notice that this new criteria is coherent with the algorithm in [4], given that it aims to find the solution which maximizes the mean power of detected symbols under the constraint of minimum length equalizer output and noise level reduction.

The covariance matrices associated to $\underline{\underline{Y}}_{t}$ and $\underline{\underline{Y}}_{0}$ are nonnegative defined and thus the SINR estimate in equation (18) corresponds to a typical Rayleigh quotient form ([10]). Therefore, it satisfies:

$\lambda_{\min }\left[\underline{\underline{Y}}_{t}^{H} \underline{\underline{Y}}_{t} ; \underline{\underline{Y}}_{0}^{H} \underline{\underline{Y}}_{0}\right] \leq \frac{\underline{\underline{E}}^{H} \underline{\underline{Y}}_{t}^{H} \underline{\underline{Y}} t \underline{\underline{E}}}{\underline{\underline{E}}^{H} \underline{\underline{Y}}_{o}^{H} \underline{\underline{Y}} \underline{\underline{E}}} \leq \lambda_{\max }\left[\underline{\underline{Y}}_{t}^{H} \underline{\underline{Y}}_{t} ; \underline{\underline{Y}}_{0}^{H} \underline{\underline{Y}}_{0}\right]$

That is, the equalizer output SINR is bounded by the minimum and maximum eigenvalues of the data matrix $\underline{Y}$ in the norm of $\underline{\underline{Y}}$. Thus, the equalizer that maximizes (18) can be obtained as the generalized eigenvector associated to the maximum generalized eigenvalue:

$$
\underline{\underline{Y}}_{t}^{H} \underline{\underline{Y}}_{t} \underline{E}=\lambda_{\max } \underline{\underline{Y}}_{o}^{H} \underline{\underline{Y}} \underline{\underline{E}} \quad \widehat{S I N R}=\lambda_{\max }
$$

As in the algorithm in [4], the new cost function is based on a deterministic criteria and, therefore, is suited to those applications where small data sets are available to estimate the equalizer weights. This new cost function integrates the information contained in $\underline{Y}_{t}$ and $\underline{Y}_{0}$. Besides, the solution $R[k]=0$ would yield a very poor SINR compared to the other solutions and, therefore, it can be rejected as a solution of the new cost function. Once the possibility of converging to this solution has been discarded, the length constraint in (12) can be released and longer equalizers can be employed. Furthermore, the exact value of the channel response length doesn't need to be known a priori. Simulations will show the performance obtained by increasing the equalizer length.

The equalizer performance can be further improved if a delay is allowed in the equalized signal. Many sets of equalizers can be obtained for different delays:

$$
\sum_{i=1}^{B} C^{i}(z) \cdot E^{i}(z)=z^{-d} \quad 0 \leq d<\operatorname{deg}\left\{C^{i}(z) \cdot E^{i}(z)\right\}
$$

yielding different delayed estimates $R(z)=z^{-d} \hat{T}(z)$. Although in average terms some delays will provide better estimates than others ([8]), the simulations performed showed that all delays are useful for noise impairment reduction due to the reduced set of data available.If the eigervalue $\lambda_{\max }$ is taken as an estimate of the SINR (see eq.(20)), it is possible to select the set of equalizers providing the best estimate of $T(z)$ by looking at the delay with greater $\lambda_{\max }$, without carrying out the full eigendecomposition for all delays. Unfortunately, this estimate is only reliable in high SNR scenarios, so in lower SNR cases the full computation of the equalizer output must be carried out to find out which delay value is preferred.

\section{RELATIONSHIP WITH OTHER ALGORITHMS}

The proposed algorithm is related to other deterministic criteria which have appeared previously in the literature such as those in [11] and [12].

The algorithm in [11] leads to the same solution as the cost function in [4] for the dual diversity case $(B=2)$ when the complex constant $\alpha=0$ and the equalizer length is selected to be the same as that one of the channel response. In this case, rather than converging to the equalizer weights, the channel response is estimated. However, this algorithm is very sensitive to the estimation of the channel response length. Unfortunately, this factor is crucial when dealing with the OFDM transmission in a frequency flat fading channel because the characterization of $C^{i}[k]$ as a short sequence is only approximated and the channel response length is not well defined. The simulations performed have shown that the performance of this algorithm is very poor in this application.

The algorithm in [4] (equations (16)-(17) ) can also be compared the deterministic method proposed in [12]. In this case, the extension of the original paper to the block transmission case will be analyzed.

It can be seen that the method proposed in [12] is based on the noise subspace of matrix $Y Y^{H}$, whereas the method in [4] was based on that one of $\underline{\underline{Y}}_{o}^{\overline{\bar{H}}} \underline{\underline{Y}}$ and the method proposed here is based on the signal subspace of $Y^{H} Y$ in the norm of $\underline{\underline{Y}}_{o}^{H} \underline{Y}_{0}$

Besides, the algorithm proposed here has a computational load much lower that one of [12], even if several values of the delay $d$ in equation (21) are used to reduce variance. Both methods have in common that they require SVD computation. However, the method proposed in this paper only one SVD must be computed and the matrix involved in it has the same size as the channel length, whereas the algorithm in [12] requires two SVD of matrices about the same size as the transmitted signal length. Since the frame duration must be chosen so that

$$
\operatorname{deg}\{T(z)\}>\operatorname{deg}\left\{C^{i}(z)\right\}
$$

in order to keep efficiency high, the computational load of the proposed algorithm is much lower than that one of [12] The dimension of the matrix involved, as well as the fact of working with the noise subspace singular vectors, has a second consequence: the algorithm in [12] is more sensitive to noise than the one proposed here.

The advantage of the method [12] in front of the one pro posed here relies in the fact that the estimate provided by the former one doesn't need to be the result of a linear equalization of the received data, whereas the one proposed here does. This means that, in principle, better results can be obtained in ill-conditioned channels where the linear equalization can have noise enhancement problems (even though in the SIMO case they are not as bad as in the single channel case $([9]))$.

Finally, it is also worth mentioning that in the multiplicative channel case, the final cost function in [4] is essentially 
the same as in [13], even though their applications as well as their derivations are different.

\section{SIMULATIONS}

Figures 3 to 7 illustrate the performance of the algorithm proposed in this paper. The plots in figures 3,6 and 7 display the percentage of realizations (500 and 1000 were averaged) for which the equalizer output EbNo was higher than the value indicated in the $\mathrm{x}$-axis. In all cases the transmitted data consisted of 128 QPSK symbols. Notice that the output EbNo depends changes on each run due to the algorithm sensitivity to the channel, data and noise realizations caused by the limited amount of data available for estimation.

Figure 3 to 6 show the performance of the algorithm in its application to an OFDM transmission in a frequency-flat fading channel corresponding to a $25 \mathrm{~Kb} / \mathrm{s}$ transmission at $1 \mathrm{GHz}$ with a mobile moving at $100 \mathrm{Km} / \mathrm{h}$ and an oversampling factor of the received signal of $135 / 128$. In this case $\mathrm{EbNo}=20 \mathrm{~dB}$. In figure 3 two antennas were used $(B=2)$. In this figure it can be observed the improvement obtained when the equalizer design criteria in (7) (I) is replaced by that one of equation (10) (II). Figures 4 and 5 depict one of the simulations averaged in figure 3-II. Notice that in this scenario the algorithm performs correctly but there is a $10 \%$ of the times for which the output EbNo is below $6 \mathrm{~dB}$. There are two reasons for that. First, the multiplicative channel does not fulfill perfectly the finite length channel hypothesis. Second, since the channel response was random, during some runs the spatial diversity was lost and the perfect equalization condition could not be achieved. Figure 4 illustrates the improvement achieved by increasing the diversity order while keeping the overall equalizer complexity constant. Notice that the \% of times for which the output $\mathrm{EbNo}<6 \%$ has greatly improved.

Figure 5 shows the performance obtained when the algorithm is applied to a TDMA burst transmission in a frequency selective channel. In this case, four antennas were simulated $(B=4)$ and channel responses were:

$$
\begin{aligned}
& \mathrm{C}^{1}(\mathrm{z})=(1+\mathrm{j})+(-0.1-0.2 \mathrm{j}) \mathrm{z}^{-1}+0.4 \mathrm{z}^{-2}+\mathrm{z}^{-3}+0.5 \mathrm{z}^{-4} \\
& \mathrm{C}^{2}(\mathrm{z})=0.1 \mathrm{j}+\mathrm{z}^{-1}-0.4 \mathrm{jz} \mathrm{z}^{-2}+0.2 \mathrm{z}^{-3}-0.5 \mathrm{z}^{-4} \\
& \mathrm{C}^{3}(\mathrm{z})=0.1+2 \mathrm{z}^{-1}-4 \mathrm{jz} \mathrm{z}^{-2}+0.2 \mathrm{z}^{-3}+\mathrm{z}^{-4} \\
& \mathrm{C}^{4}(\mathrm{z})=(1+0.8 \mathrm{j})-2 \mathrm{j} \mathrm{z}^{-1}-0.4 \mathrm{j} \mathrm{z}^{-2}+0.2 \mathrm{z}^{-3}+(1-0.5 \mathrm{j}) \mathrm{z}^{-4}
\end{aligned}
$$

This figure illustrates the improvement obtained by increasing the equalizer length. In this case, $\mathrm{EbNo}=15 \mathrm{~dB}$ and the equalizers of length 2 (I) and 4 (II) were designed using equation (10).

\section{CONCLUSIONS}

In this paper a new cost function has been proposed for blind equalization of mobile channels in diversity receivers. The deterministic criteria used for equalizer design has been shown to work well for short sets of data, both in frequency selective and frequency flat fading channels. The algorithm can be applied with long equalizers and is well suited to its application in OFDM transmission in multiplicative channels, since it requires no redundancy introduction and it is robust to all application constraints: new channel estimation for each transmitted frame and not-known channel length. The relationship with other blind algorithms has also been explored.

\section{REFERENCES}

[1] R.Steele, Mobile Radio Communications, Pentech Press, 1992

[2] B.LeFloch et al, 'Coded Orthogonal Frequency Division Multiplex', Proc. of IEEE, June 1995

[3] M.Lamarca, G.Vázquez, 'Transform modulations in mobile communications', To be published in Proc. of PIMRC'97, Helsinki (Finland), September 1997

[4] M.Lamarca, G.Vázquez, Multichannel receivers for OFDM and TDMA in mobile communications', Proc. of ICASSP'97, April 1997, Munich (Germany)

[5] M.Lamarca and G.Vázquez, 'Channel estimation for transform modulations in mobile communications', Proc. of EUSIPCO'96, Trieste (Italy), Sept. 1996

[6] T.Kailath, Linear Systems, Prentice-Hall, 1980

[7] Y.Li, Z.Ding, 'Blind channel identification based on second order cyclostationary statistics', Proc. of ICASSP'93, Minneapolis (USA)

[8] D.Gesbert, P.Duhamel, S.Mayrargue, 'Blind multichannel adaptive MMSE equalization with controlled delay', Proc. of $8^{\text {th }}$ IEEE Signal Processing Workshop on SSAP, Corfu (Greece), 1996

[9] G.Giannakis, S.D.Halford, 'Blind fractionally-spaced equalization of noisy FIR channels: adaptive and optimal solutions', Proc. of ICASSP'95, Detroit (USA)

[10] G.H.Golub, C.F.Van Loan, Matrix Computations, Johns Hopkins Univ. Press, 1989

[11] G.Xu et al, 'A Least-Squares Approach to Blind Channel Identification', IEEE Trans. on Signal Processing, Vol.43, No.12, December 1995

[12] H.Liu, G.Xu, 'Closed-form blind symbol estimation in digital communications', IEEE Trans. on Signal Processing, Vol.43, No.11, November 1995

[13] M.Courville, P.Duhamel, P.Madel and J.Palicot, 'Blind equalization of OFDM systems based on the minimization of a quadratic criterion', Proc. of ICC'96

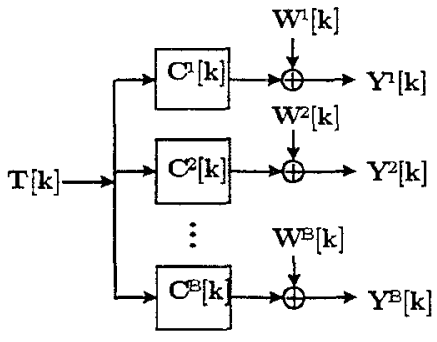

(a)

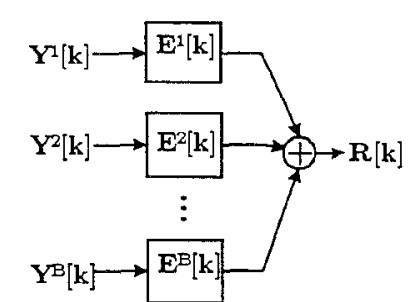

(b)
Fig.1. Block diagram of the multichannel system. (a) Transmission; (b) Equalization. 

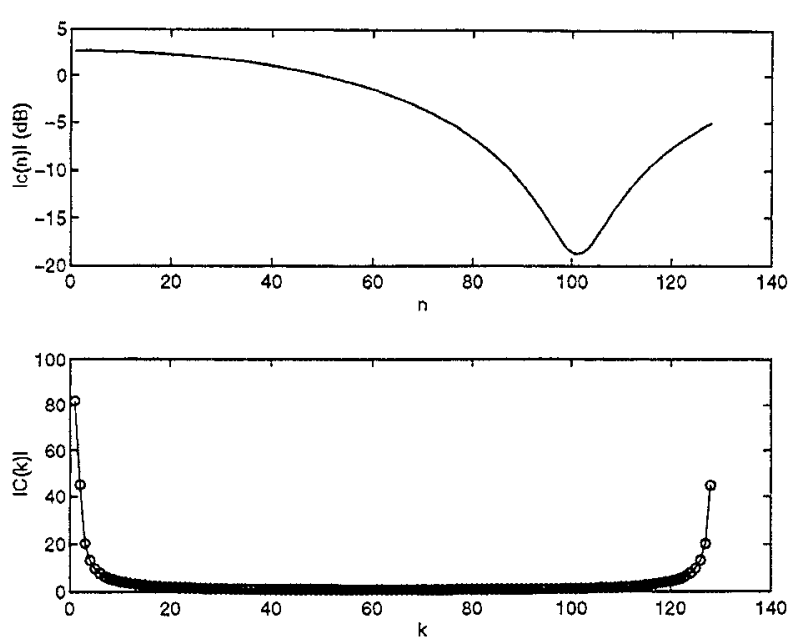

Fig.2. Typical realization of the Rayleigh fading channel $c[n]$ and its DFT $C[k]$ using the same parameters as the simulations in section 6 .

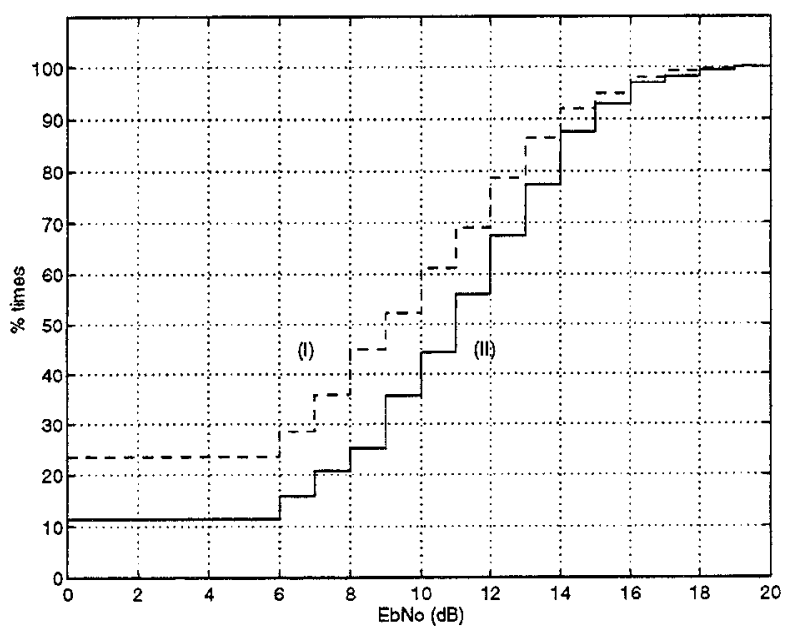

Fig.3. Algorithm performance in the transmission of a OFDM signal in a Rayleigh frequency-flat fading scenario. Comparison of the two cost functions.
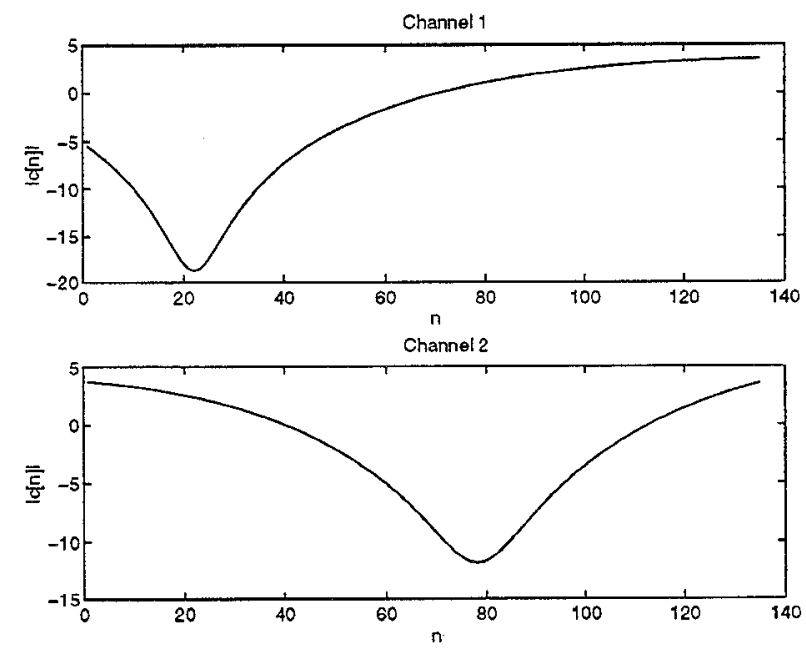

Fig.4. Time evolution of the Rayleigh channels corresponding to the equalizer output in figure 5 .

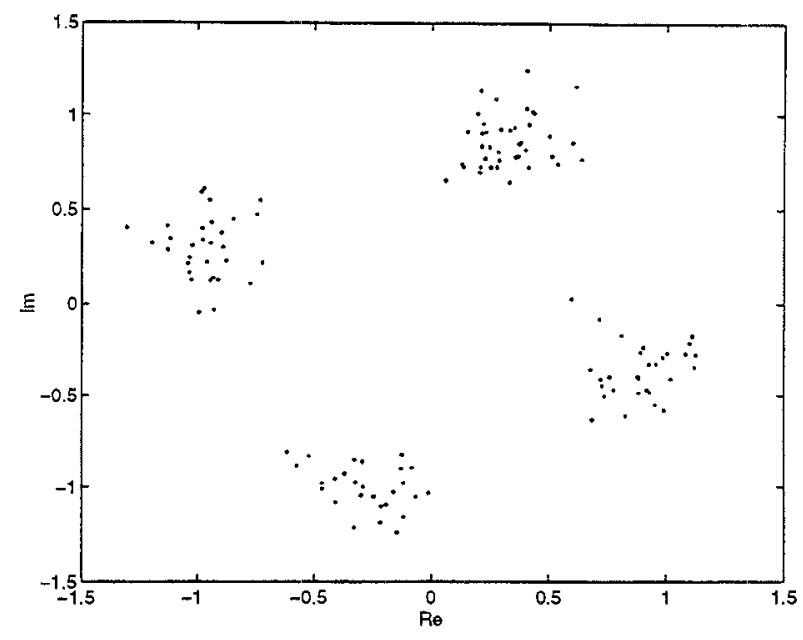

Fig.5. Equalizer output constellation for the channels in figure 4.

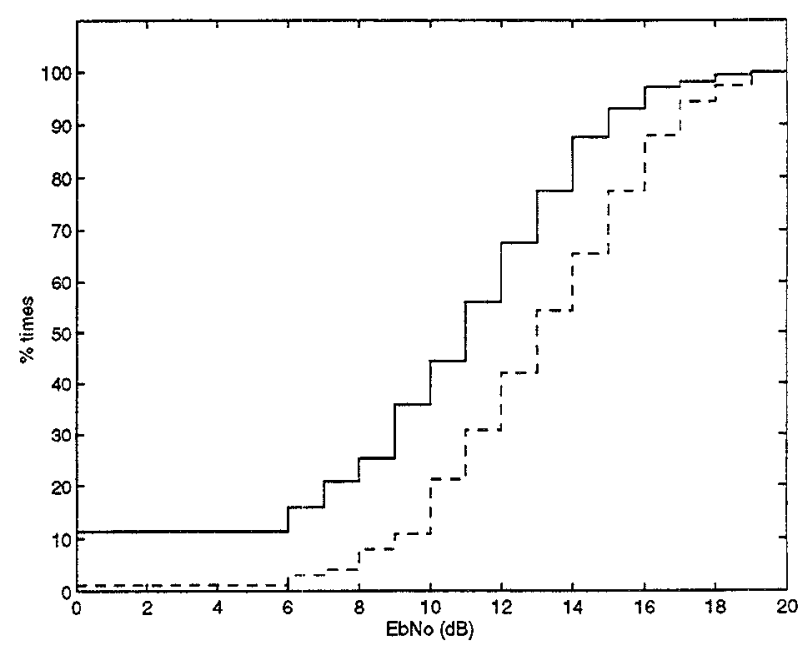

Fig.6. Algorithm perfomance in the transmission of an OFDM signal in a frequency flat fading scenario. Comparison of $B=2$ and equalizer length $=3$ (solid line) with $B=3$ and equalizer length $=2$ (dashed line).

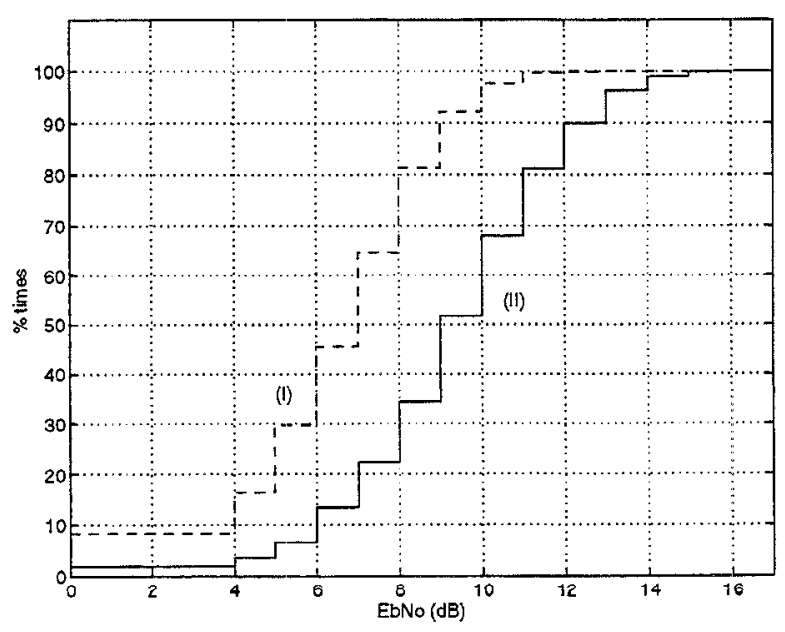

Fig.7. Algorithm performance in the transmission of a TDMA signal in a frequency-selective channel. 